\title{
Quantitative Analysis of Enlarged Cervical Lymph Nodes with Ultrasound Elastography
}

\author{
Jun-Peng Zhang, Hua-Yan Liu, Chun-Ping Ning, Jing Chong, Yong-Mei Sun*
}

\begin{abstract}
Purpsoe: To investigate the diagnostic value of quantitative analysis of a tissue diffusion and virtual touch tissue imaging quantification (VTIQ) technique with acoustic radiation force impulse (ARFI) elastography for assessing enlarged cervical lymph nodes. Materials and Methods: Fifty-six enlarged cervical lymph nodes confirmed by pathologic diagnoses were covered in the study. According to the results of pathologic diagnosis, patients were classified into benign and malignant groups. All the patients were examined by both conventional ultrasonography and elastography. AREA\% and shear wave velocity (SWV) in ROI of different groups were calculated and compared using ROC curves. Cut-off points of AREA\% and SWV were determined with receiver operating characteristic curves. Results: Final histopathological results revealed 21 cases of benign and 35 cases of malignant lymph nodes. The mean values of AREA\% and SWV in benign and malignant groups were $45.0 \pm 17.9 \%$ and $2.32 \pm 0.57 \mathrm{~m} / \mathrm{s}$, and $61.3 \pm 21.29 \%$ and $4.36 \pm 1.25) \mathrm{m} / \mathrm{s}$, respectively. For the parameters of elastography, "AREA\%" and SWV demonstrated significant differences between groups $(p=0.002)$. AREA\% was positively correlated with $S W V$ with a correlation coefficient of $0.809(P<0.001)$. Conclusions: Stiffness of different lymph node diseases in patients may differ. Elastography can evaluate changes sensitively and provide valuable information to doctors. The study proved that the VTIQ elastography technique can play an important role in differential diagnosis of lymph nodes.
\end{abstract}

Keywords: Elasticity imaging techniques - virtual touch tissue imaging - lymphadenopathy

Asian Pac J Cancer Prev, 16 (16), 7291-7294

\section{Introduction}

The differentiation of malignant from benign lymph nodes has important significance as it is a common situation faced by clinicians. Conventional ultrasound was the preferred screening method of lymph nodes although with low specificity. Since the clinical application of ultrasound elasticity,reflecting the fact of tissue stiffness at many sites to differentiate malignant from benign lesions in the breast, thyroid, liver, etc. In addition, a few preliminary reports suggest that this technique was positively correlated with histopathological reasults (Klotz et al., 2014; Ying Shi et al., 2014; Han et al., 2015). Currently the application of quantitative elasticity techniques include elastic tissue diffusion quantitative and acoustic radiation force impulse imaging technology (Dawood et al., 2014; Fu et al., 2014). The main objective of was to assess utility of elastic diffusion quantitative comparing ARFI for improved lymph node evaluation.

\section{Materials and Methods}

\section{Patients}

From August 2014 to April 2015, 56 cervical lymph nodes in 56patients underwent conventional gray-scale and US elastgraphy. The study group comprised of 25males and 31 females (mean age 49.6 \pm 14 . 6years, range 17-77 years). Both conventional ultrasound imaging and real-time ultrasoundelastography were done by using linear probe. Sonographic features of swelling lymph nodes were documented. The final diagnosis of the lymph nodes was determined by histopathological examination.

\section{Real time elastography (RTE)}

RTE measurements were performed by an Hitachi Preirus (Hitachi Medical Corporation, Tokyo, Japan) with 7.5-13. 0MHz linear array probes. Patient and transducer positioning were similar to that of conventional sonography. The patient was asked to avoid swallowing and hold their breath during the measurement. Images were obtained by applying light repetitive compression at the skin above the object lymph nodes. The sampling frame was usually two-three times of the lesion including subcutaneous fat, and the longus colli muscle (Fu et al., 2014). In our experience, the ROI should exceed the lymph nodes boundaries at least $5 \mathrm{~mm}$ on each side. It was important to avoid the presence of bone or blood vessels in the scan area. The most representative image obtained with optimal compression factor was stored for further assessment. Then the radiologist choose the strain histogram and draw a largest rectangle within lymph node, the calculation of the AREA\% of elastogram will 
be offered by the integrated system. At least 5 groups of parameters will be used for quantitative analysis.

\section{Virtual touch imaging quantification (VTIQ)}

VTIQ measurements were performed by an Acuson S3000 ultrasound system (Siemens Medical Solutions) using a linear array transducer with bandwidth of 4-9 MHz. For VTIQ measurement, the patients'position was similar with conventional ultrasonography examination. A ROI is placed and acoustic push pulses were applied across the ROI. Then stiffness shown by a color-coded two-dimensional shear wave is immediately displayed in the ROI on the screen. VTIQ evaluation includes four patterns: quality, time, displacement, velocity. We did not employ all kinds of patterns for assessing VTIQ. The predominant colour coded VTIQ patterns are quality and velocity patterns. After obtaining a static and quality map (good-green, marginal-yellow, poor-red) image for analysis. The velocity of lymph nodes were measured. In each measured area we obtained five-six measurements to achieve mean value of VTIQ.

All the ultrasound examinations were performed by the same radiologist with experience of more than 5-years of ultrasound scanning.

\section{Statistical analysis}

All analyses were performed using the software SPSS 20.0. The level of significance was set at 0.05 for all tests. All quantitative data are expressed by mean \pm S. D. We used the chi-square test to compare categorical variables. Oneway analysis of variance (One-Way ANOVA) was used for comparison among groups. Spearman rank correlation analysis was used for correlation analysis.

\section{Results}

\section{General features}

There were 56 lymph nodes included in our group of which 21 were benign (15 cases of non specific lymphadenitis and 6 cases of Tuberculous) and 31 were malignant (18 adenocarcinomas, 10squamous carcinomas, 5 papillary thyroid carcinomas and 2 small cell carcinoma).

\section{Conventional ultrasound features}

$\mathrm{L} / \mathrm{S}$, absent of echogenic hilum and RI between groups were statistically significant. There was no significant difference between groups as for the number of lymph nodes per patient, and presence of necrosis (Table 1).

\section{Elastography ultrasound features}

The mean values of AREA\% and VTIQ of the benign and malignant $1 y m p h$ nodes were $(45.03 \pm 17.85) \%$, $(2.32 \pm 0.57) \mathrm{m} / \mathrm{s}, 61.31 \pm 21.29 \%, 4.36 \pm 1.25(\mathrm{~m} / \mathrm{s})$ respectively, showing statistical differences (Table 2). One patient with incorrect diagnosis on RTE got correct diagnosis afterARFI examination. ROC curves were constructed in order to determine the best cutoff value of AREA\% and SWV malignant lymph nodes from benign nodes. A lymph nodes AREA\% of $45.27 \%(p<0.05)$ was associated with $85.7 \%$ sensitivity and $62 \%$ specificity and an area under the curve (AUC) equal to 0.731. For lymph nodes shear wave velocity, the optimal cut-off for clinical decision making concerning a low false positive rate was chosen to be $3.14 \mathrm{~m} / \mathrm{s}$. This cut-off yielded a sensitivity of $77.1 \%$ and a specificity of $85.7 \%$ (Figure 1 ).

Table 1. Ultrasonographic Findings Correlated with Pathologic Diagnosis in 56 Cervical Lymph Nodes

\begin{tabular}{lrrr}
\hline Characteristics & $\begin{array}{r}\text { Malignant } \\
\text { group }\end{array}$ & $\begin{array}{r}\text { Benign } \\
\text { group }\end{array}$ & P value \\
\hline Single/multiple & $6 / 29$ & $0 / 21$ & 0.07 \\
Absent hilus (Y/N) & $4 / 31$ & $8 / 13$ & 0.04 \\
Necrosis (Y/N) & $4 / 31$ & $5 / 16$ & 0.27 \\
$\mathrm{~L} / \mathrm{S}(\geq 2 /<2)$ & $5 / 30$ & $10 / 11$ & 0.01 \\
Resistance index $(\leq 0.7 />0.7)$ & $15 / 20$ & $18 / 3$ & 0.00 \\
\hline
\end{tabular}

Table 2. Elastography Findings Correlated with Pathologic Diagnosis in 56 Cervical Lymph Nodes

\begin{tabular}{lccc}
\hline Parameters & $\begin{array}{c}\text { Malignant } \\
\text { group }\end{array}$ & $\begin{array}{c}\text { Benign } \\
\text { group }\end{array}$ & P \\
\hline SR $(\leq 2.40 />2.40)$ & $6 / 29$ & $17 / 4$ & 0.00 \\
AREA\%( $\leq 45.27 />45.27)$ & $4 / 31$ & $16 / 5$ & 0.00 \\
SWV $(\leq 3.14 />3.14)$ & $5 / 30$ & $16 / 5$ & 0.00 \\
\hline
\end{tabular}

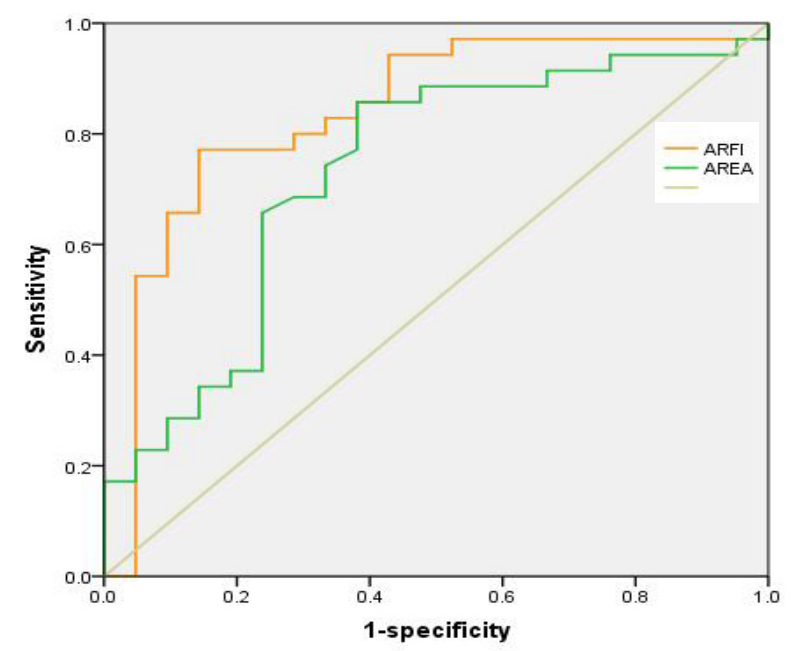

Figure 1. Receiver Operating Characteristic Curve for AREA \%, ARFI

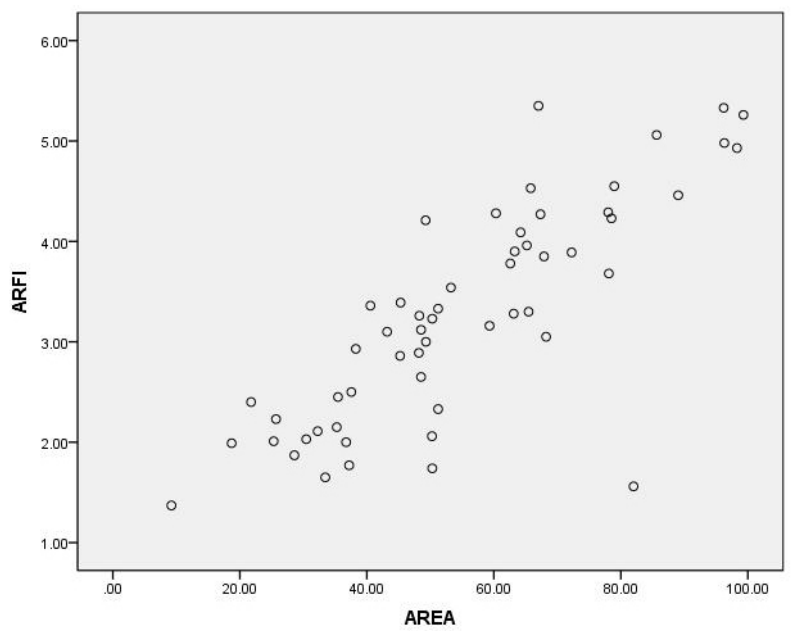

Figure 2. The Interclass Correlation Coefficient between AREA \% and VTIQ. The interclass correlation coefficient between AREA\% and VTIQ values was 0.809 . 


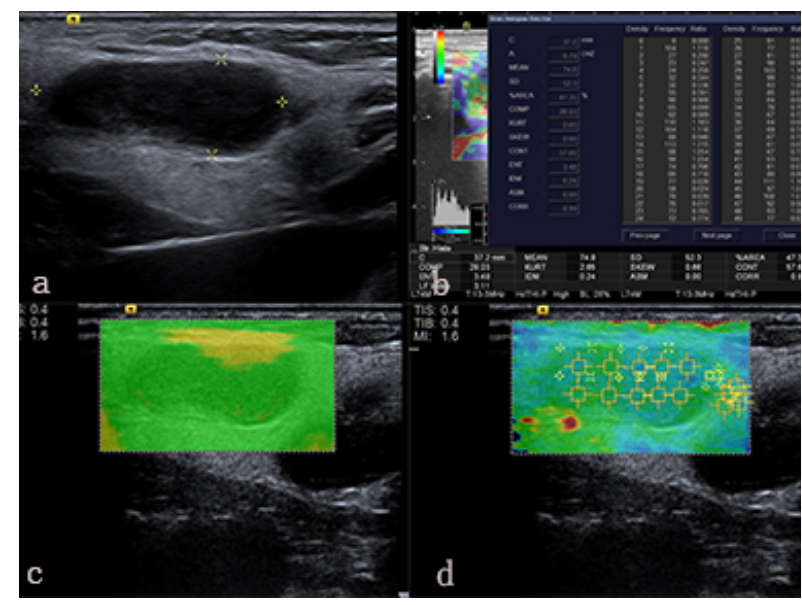

Figure 3. A Woman of 64 Years Old, with Cervical Lymphadenopathy. (a) Enlarged lymph nodes in level IV of the right neck. It was with equivocal diagnosis on conventional US. (b). Elastographic image on the right shows the AREA\% of LN. (c). Most of the lymph node is colored in green, as an indicator for a high quality of generated shear waves.(d) VTIQ velocity colour overlay displayed relative shear wave velocities according to the adjacent colour spectrum



Figure 4. A woman of 32 Years Old, with Cervical

Lymphadenopathy. (a) Enlarged lymph nodes in level IV of the right neck. proved to be malignant lymph node. (b). Elastographic image on the right shows the AREA\% of LN. (c). Most of the lymph node is colored in green, as an indicator for a high quality of generated shear waves.(d) VTIQ velocity colour overlay displayed relative shear wave velocities according to the adjacent colour spectrum.

\section{The interclass correlation coefficient between VTIQ and} AREA\%

The 56 numeric VTIQ values were compared with the corresponding 56 AREA\% values. The interclass correlation coefficient between VTIQ and AREA\% was 0.809 (Figure 2).

\section{Discussion}

The status of lymph node is one of the most important predictors of prognosis of head and neck cancers. The reasons of enlarged cervical Lymph nodes included reactive hyperplasia,lymphoma, lymphadenitis, granuloma, metastasis, tuberculosis, etc (Dudea, 2013; Fujiwara et al., 2013; Hefeda and Badawy, 2014). Generally, it could be divided into benign and malignant enlargement. Correct diagnosis the nature of enlarged lymph nodes is extremely important because it directly affects the prognosis and treatment decision (Kau et al., 2000). Ultrasonography (US) was used as a first line diagnostic means . However, specific criteria for different lymph nodes are still controversial. One of the important diagnostic method for lymph nodes is fine needle aspiration biopsy (FNAB), although it is reliable and relatively safe, it may lead to complications and false negative (Reddy et al., 2015).

RTE is the commonest technique that provides information about the relative stiffness of the structures within the scan plane, inside a region of interest. Now, the measurement methods of RTE include patterns, scores or grades,which were semi-quantitative (Teng et al., 2012; Lo and Liao, 2014). These has led to considerable confusion about the findings, more operator-dependency, poor reproducibility and consistency in different studies (Lo and Liao, 2014). The quantitative analysis of tissue diffusion was a new generation of RTE technology equipped with quantitative analysis of tissue diffusion. The new technology used quantitative software integrated into the Hitachi system which provide 10 parametres of selected area. In our study, we used a quantitative parametre AREA\% which the software provide. This parametreswas has been already used in assessment of liver stiffness in patients with chronic hepatitis, Hashimoto thyroiditis (Ying Shi et al., 2014; Fang Shi-bao et al., 2015).

Virtual touch tissue quantification (VTQ) represents a traditional technology of acoustic radiation force impulse (ARFI) imaging, which calculate the horizontal shear wave speed generated by the longitudinal pulse generated from the transducer. It is calculated and expressed in the unit "meters/second" $(\mathrm{m} / \mathrm{s})$, the faster the shearwave propagates means the tissue is more stiffer. Virtual touch tissue imaging quantification (VTIQ) is a new elastography technology based on VTQ. The VTIQ technique does not rely on the operator's ability to apply appropriate mechanical pressure to the tissue. It also displays tissue stiffness on a two-dimensional color coded map,additional qualitative maps like the displacement, time and quality are available. VTIQ had some other advantages :the smaller ROI ( $1 \times 1 \mathrm{~mm})$, easier to measure objective and measureable range up to $10 \mathrm{~m} / \mathrm{s}$ (Golatta et al., 2014; Ianculescu et al., 2014; Takashi Matsuzuka et al., 2015; Tang Li et al., 2015).

In our study, elastography ultrasound imaging revealed higher AREA\% and SWV for malignant lymph nodes than benign lymph nodes (Figure 3,4). The cut-off values of AREA\% and VTIQ of benign and malignant lymph nodes were $45.27 \%, 3.14 \mathrm{~m} / \mathrm{s}$, corresponding with $85.7 \%, 77.1 \%$ sensitivity and $62.0 \%, 85.7 \%$ specificity,respectivly. The stiffness of the lymph node is closely related to the biological characteristics. Malignant lymph nodes offen be infiltrated by tumour cells, necrosis, calcification. Benign lymph nodes, not containing metastatic deposits, have the similar stiffness to the neck normal tissue. According to this theory, the malignant lymph nodes were mostly stiffer than benign (Bhatia et al., 2010; Tan et al., 2010; Cosgrove et al., 2013; Fodor, 2013). Statistical analysis reveal that area under the ROC curve of AREA\%, VTIQ was 0.731,0. 
838 , respectively. The specificity of VTIQ is $85.7 \%$, larger than AREA\%, due to small ROI. One tuberculo-lymph node was misdiagnosed as malignant. In this case,more coarse calcification in node without necrosis and abscess ,neither RTE elastography nor VTIQ avoid that. The histopathology of T. B on ultrasonography was classified into four types: acute inflammation type, cheesy necrosis type, cold abscess type and concrescent calcification type. The tissue tiffer of T.B. is complexity, is always the diagnostic dilemma between T. B. and malignant disease of LN (Geng-chen, et al., 2013).

There are some limitations in our study. Only a few of lymph nodes were studied. Further studies with a greater variety of lymph nodes will be important. Lymphoma was excluded in this study because it might be softer than metastatic LNs (Ma et al., 2014; Okasha et al., 2014).

In Conclusion,elastography with two kinds of quantitative Evaluation technical implementations seems to be promising tools that can provide additional stiffness information of cervical lymphadenopathy for unknown reason. For simplification purposes, we suggest using the VTIQ to qualitative assment lymph nodes in the future. More work is still needed to fully explore the clinical values of VTIQ. It may open new perspectives assessment of cervical lymphadenopathy.

\section{References}

Bhatia KS, Cho CC, Yuen YH, et al (2010). Real-time qualitative ultrasound elastography of cervical lymph nodes in routine clinical practice: interobserver agreement and correlation with malignancy. Ultrasound Med Biol, 36, 1990-7.

Cosgrove D, Piscaglia F, Bamber J, et al (2013). EFSUMB guidelines and recommendations on the clinical use of ultrasound elastography. Part 2: Clinical applications. Ultraschall Med, 34, 238-53.

Dudea SM (2013). Differentiating benign from malignant superficial lymph nodes with sonoelastography. Med Ultrasonography, 15, 132-9.

Dawood HA, Hassan TA, Mohey N (2014). Value of combined real time sonoelastography and apparent diffusion coefficient value measurement in differentiation of enlarged neck lymph nodes. Egyptian J Radiol Nuclear Med, 45, 387-94.

Fodor D (2013). The utility of elastography and CEUS for the differentiation between benign and malignant cervical lymphadenopaty. Three cases report. Med Ultrasonography, 15, 63-6.

Fujiwara T, Tomokuni J, Iwanaga K, et al (2013). Acoustic radiation force impulse imaging for reactive and malignant/ metastatic cervical lymph nodes. Ultrasound Med Biol, 39, 1178-83.

Fu Y, Shi Y-F, Yan K, et al (2014). Clinical Value of Real Time Elastography in Patients with Unexplained Cervical Lymphadenopathy: Quantitative Evaluation. Asian Pac J Cancer Prev, 15, 5487-92.

Fang Sb, Wang YY, Ning CP, et al (2015). Value of quantitative study of tissue diffusion for assessment of hardness of thyroid tissue in patients with hashimoto thyroiditis of different functional statu. pdf. Acta Acad Med Qingdao Universitatis, 51, 8-10.

Golatta M, Schweitzer-Martin M, Harcos A, et al (2014). Evaluation of virtual touch tissue imaging quantification, a new shear wave velocity imaging method, for breast lesion assessment by ultrasound. Biomed Res Int, 960962,1-7.
Hefeda MM, Badawy ME (2014). Can ultrasound elastography distinguish metastatic from reactive lymph nodes in patients with primary head and neck cancers? Egyptian J Radiol Nuclear Med, 45, 715-22.

Han R, Li F, Wang Y, et al (2015). Virtual touch tissue quantification (VTQ) in the diagnosis of thyroid nodules with coexistent chronic autoimmune Hashimoto's thyroiditis: a preliminary study. Eur J Radiol, 84, 327-31.

Kau RJ, Alexiou C, Stimmer H, et al (2000). Diagnostic procedures for detection of lymph node metastases in cancer of the larynx. ORL J Otorhinolaryngol Relat Spec, 62, 199-203.

Klotz T, Boussion V, Kwiatkowski F, et al (2014). Shear wave elastography contribution in ultrasound diagnosis management of breast lesions. Diagn Interv Imaging, 95, 813-24.

Lanculescu V, Ciolovan LM, Dunant A, et al (2014). Added value of Virtual Touch IQ shear wave elastography in the ultrasound assessment of breast lesions. Eur J Radiol, 83, 773-7.

Lo WC, Liao LJ (2014). Comparison of Two Elasticity Scoring Systems in the Assessment of the Cervical Lymph Nodes. $J$ Med Ultrasound, 22, 140-4.

Ma B, Jia Y, Wang Q, et al (2014). Ultrasound of primary thyroid non-Hodgkin's lymphoma. Clin Imaging, 38, 621-6.

Okash HH, Mansour M, Attia KA, et al (2014). Role of high resolution ultrasound/endosonography and elastography in predicting lymph node malignancy. Endosc Ultrasound, 3, 58-62.

Reddy DL,Venter WD,Pather S (2015). Patterns of Lymph Node Pathology; Fine Needle Aspiration Biopsy as an Evaluation Tool for Lymphadenopathy: A Retrospective Descriptive Study Conducted at the Largest Hospital in Africa. PLoS One, 10, 130-48.

Shi Y,Wang XH,Zhang HH, et al (2014). Quantitative analysis of real-time tissue elastography for evaluation of liver fibrosis. pdf. Int J Clin Exp Med, 7, 1014-21.

Tan R, Xiao Y, He Q (2010). Ultrasound elastography: Its potential role in assessment of cervical lymphadenopathy. Acad Radiol, 17, 849-55.

Teng DK,Wang H,Lin YQ,et al (2012). Value of Ultrasound Elastography in Assessment of Enlarged Cervical Lymph Nodes. Asian Pac J Cancer Prev, 13, 2081-5.

Takashi M, Masahiro S, Satoshi S, et al (2015). Stiffness of salivary gland and tumor measured by new ultrasonic techniques Virtual touch quantification and IQ. pdf. Auris Nasus Larynx, 42, 128-33.

Tang L, Xu HX, Li JW,et al (2015). Novel technique of virtual touch tissue imaging quantification shear wave elastography in differential diagnosis of thyroid nodules: initial experience. Chin J Med Ultrasound, 12, 241-6.

Zhang GC,Li JL,Cao BS, et al (2013). Ultrasound characteristic of cervical tuberculous lymphadenitis correlating with pathology. Chinese J Ultrasound Med, 29, 879-82. 